\title{
Time-delayed contrast-enhanced MRI improves detection of brain metastases and apparent treatment volumes
}

\author{
Marina Kushnirsky, BA, ${ }^{1}$ Vinh Nguyen, MD, ${ }^{2}$ Joel S. Katz, DO, ${ }^{3}$ Jared Steinklein, MD, ${ }^{2}$ \\ Lisa Rosen, ScM, ${ }^{4}$ Craig Warshall, MD, ${ }^{2}$ Michael Schulder, MD, ${ }^{3}$ and Jonathan P. S. Knisely, MD ${ }^{5}$ \\ ${ }^{1}$ Hofstra North Shore-LIJ School of Medicine at Hofstra University, Hempstead; ${ }^{2}$ Department of Radiology, North Shore-LIJ \\ Health System, New Hyde Park; ${ }^{3}$ Department of Neurosurgery, North Shore-LIJ Health System, Lake Success; ${ }^{4}$ North Shore-LIJ \\ Feinstein Institute for Medical Research, Manhasset; and ${ }^{5}$ Department of Radiation Medicine, North Shore-LIJ Health System, \\ Lake Success, New York
}

OBJECTIVE Contrast-enhanced MRI is the preeminent diagnostic test for brain metastasis (BM). Detection of BMs for stereotactic radiosurgery (SRS) planning may improve with a time delay following administration of a high-relaxivity agent for 1.5-T and 3-T imaging systems. Metastasis detection with time-delayed MRI was evaluated in this study.

METHODS Fifty-three volumetric MRI studies from 38 patients undergoing SRS for BMs were evaluated. All studies used 0.1-mmol/kg gadobenate dimeglumine (MultiHance; Bracco Diagnostics) immediately after injection, followed by 2 more axial T1-weighted sequences after 5-minute intervals (final image acquisition commenced 15 minutes after contrast injection). Two studies were motion limited and excluded. Two hundred eighty-seven BMs were identified. The studies were randomized and examined separately by 3 radiologists, who were blinded to the temporal sequence. Each radiologist recorded the number of BMs detected per scan. A Wilcoxon signed-rank test compared BM numbers between scans. One radiologist determined the scan on which BMs were best defined. All confirmed, visible tumors were contoured using iPlan RT treatment planning software on each of the $3 \mathrm{MRI}$ data sets. A linear mixed model was used to analyze volume changes.

RESULTS The interclass correlations for Scans 1, 2, and 3 were $0.7392,0.7951$, and 0.7290 , respectively, demonstrating excellent interrater reliability. At least 1 new lesion was detected in the second scan as compared with the first in $35.3 \%$ of subjects $(95 \% \mathrm{Cl} 22.4 \%-49.9 \%)$. The increase in BM numbers between Scans 1 and 2 ranged from 1 to 10. At least 1 new lesion was detected in the third scan as compared with the second in $21.6 \%$ of subjects $(95 \% \mathrm{Cl}$ 11.3\%-35.3\%). The increase in BM numbers between Scans 2 and 3 ranged from 1 to 9 . Between Scans 1 and 3, additional tumors were seen on $43.1 \%$ of scans (increase ranged from 1 to 14). The median increase in tumor number for all comparisons was 1. There was a significant increase in number of BMs detected from Scan 1 to Scan $2(p<0.0367)$ and from Scan 1 to Scan $3(p<0.0264)$. In 34 of the 51 subjects $(66.7 \%)$, the radiologist selected the third scan as the one providing the clearest tumor definition. There was an average $25.4 \%$ increase in BM volume between Scans 1 and 2 ( $p<$ $0.0001)$ and a $9 \%$ increase in BM volume between Scans 2 and $3(p=0.0001)$.

CONCLUSIONS In patients who are being prepared for SRS of BMs, delayed MRI after contrast injection revealed more targets that needed treatment. In addition, apparent treatment volumes increased with a time delay. To avoid missing tumors that could be treated at the time of planned SRS and resultant "treatment failures," the authors recommend that postcontrast MR images be acquired between 10 and 15 minutes after injection in patients undergoing SRS for treatment of BMs.

http://thejns.org/doi/abs/10.3171/2015.2.JNS141993

KEY WORDS brain metastases; delayed MRI; gadobenate dimeglumine; oncology

$\mathrm{B}$ RAIN metastases (BMs) are the most common intracranial tumors in adults, occurring in $20 \%-40 \%$ of cancer patients. ${ }^{26}$ Their incidence has been increasing due to both prolonged systemic control and improved detection, making BMs a growing cause of mortality and morbidity. ${ }^{14,31}$ Magnetic resonance imaging is currently the standard of care for the diagnosis of BMs because its sensitivity is superior to $\mathrm{CT}, 2,32$ and refinements in MRI techniques allow the modality to detect metastases that are not seen with routine image-acquisition protocols. The

ABBREVIATIONS BM = brain metastasis; GRE = gradient echo; $\mathrm{SRS}=$ stereotactic radiosurgery .

SUBMITTED September 19, 2014. ACCEPTED February 4, 2015.

INCLUDE WHEN CITING Published online September 11, 2015; DOI: 10.3171/2015.2.JNS141993. 
detection of BMs, even when they are tiny, is essential for optimizing treatment, providing cost-effective care, and preserving a good quality of life among patients with metastatic disease.

Advances in surgery, systemic therapies, and radiosurgery have significantly enhanced local control and survival for patients with BMs, even for those with multiple metastases..$^{1,934}$ However, these treatments rely on proper tumor visualization. Tumor detection is particularly important for stereotactic radiosurgery (SRS), a noninvasive focal treatment that can target and control metastases while avoiding whole-brain irradiation. Stereotactic radiosurgery does not cause the long-term neurological deficits associated with whole-brain irradiation. ${ }^{2,4,5,10,12}$ However, because SRS is a local therapy, it is important to avoid "missing" or inadequately treating metastases that could be detected and targeted during planned SRS.

Furthermore, published guidelines that were developed to assist with the selection of which treatment modality to use for patients with BMs (surgery, whole-brain irradiation, SRS alone or as a postresection adjuvant therapy, or palliative care) depends, among other things, on factors such as the number of BMs and their sizes or volumes. ${ }^{18,20,25,28,29}$ Improving radiological imaging methods is instrumental for correctly ascertaining the size and number of lesions for resection and radiation treatment calculations. ${ }^{20}$ In addition, the current prognostic characteristics of individual patients rely on recursive partitioning analysis, for which accurate imaging is essential. ${ }^{28}$ Importantly, BM number has been shown to assist with survival prognostication for certain tumor histologies. ${ }^{28}$

The aim of this study was to determine whether BM detection for SRS planning improved with a time delay following administration of gadobenate dimeglumine (MultiHance; Bracco Diagnostics) for 1.5-T and 3-T imaging systems. The high relaxivity of gadobenate dimeglumine provides an increased lesion-to-brain signal intensity on T1-weighted MR images, resulting in increased contrast enhancement. Therefore, there is support for its use at lower doses than other contrast agents. ${ }^{21}$

This is the first study to examine the use of gadobenate with time-delayed isotropic imaging. Previous studies have examined time delays for other intracranial pathologies, ${ }^{7,24}$ as well as for brain tumors when using other contrast agents. ${ }^{6,32}$ Some have suggested the use of double- or triple-dose contrast to improve BM detection, $8,30,35$ but this may increase the risk of developing complications (e.g., nephrogenic systemic fibrosis in patients with renal impairment ${ }^{13}$ ) and adds to the cost of the examination. Although multiple-dose, gadolinium-enhanced imaging is certainly feasible when a dedicated SRS-planning MRI is scheduled, it is not feasible for routine surveillance or diagnostic imaging for patients who are known or suspected to have BMs.

\section{Methods \\ Patients}

This research study recruited patients over the age of 18 with a Karnofsky Performance Scale score of 70 or above, ${ }^{11}$ who previously had a contrast-enhanced MRI that confirmed 1-4 BMs. Thirty-eight patients with a mean age of 64 years ( 23 female, 15 male) gave informed consent. Fifty-three pre- and post-SRS MRI studies were conducted, 2 of which were motion limited and excluded. Seventeen scans were obtained prior to any SRS, and 36 were obtained as surveillance imaging studies in patients who had previously undergone SRS. No patient had previously undergone whole-brain irradiation. This research study was approved by the North Shore-LIJ Health System institutional review board. Lung cancer was the primary site of disease for 23 patients, breast cancer for 6 , melanoma for 4 , colon cancer for 2 , renal cancer for 2 , and ovarian cancer for 1 (Table 1).

\section{MRI}

Imaging was performed on either a 3-T (Verio 3T, Siemens or Signa HDxt 3.0T, GE Healthcare) or 1.5-T (Signa HDxt 1.5T, GE Healthcare) MRI system. The protocol included an initial isotropic T1-weighted gradient echo (T1-GRE) noncontrast sequence lasting 4-5 minutes. Then, standard $(0.1 \mathrm{mmol} / \mathrm{kg})$ weight-based dosing of gadobenate dimeglumine was administered followed by a $20-\mathrm{ml}$ saline flush. The time of the contrast injection was recorded for all patients. Contrast administration via power injection at $2 \mathrm{ml} / \mathrm{sec}$ was immediately followed by an axial isotropic T1-GRE postcontrast study 4-5 minutes in duration. The third scan was an axial T2-weighted study lasting 5-6 minutes. Two more axial isotropic T1GRE postcontrast scans were sequentially obtained. In total, each patient received 3 axial T1-weighted postcontrast MRI scans. This protocol was designed to meet the time limit of a 30-minute study.

The isotropic T1-GRE imaging parameters were BRAVO (GE 1.5T) T1-weighted (TR/TE/TI 10.7/4.4$14 / 450 \mathrm{msec}$, flip angle $13^{\circ}$ ) with $1.0-\mathrm{mm}$ slice thickness, bandwidth $25 \mathrm{kHz}$, matrix $320 \times 320$, FOV 240 $\mathrm{mm}$. We also used MPRAGE (Siemens 3T) (TR/TE/ TI 1900/3.11/900 msec, flip angle $9^{\circ}$ ) with $0.9-\mathrm{mm}$ slice thickness.

\section{Image Analysis}

When the study was complete, each of these scans was recorded and assigned to either a first, second, or third imaging group. The studies were randomized and examined separately by 3 radiologists, who were blinded to the temporal sequence. Each radiologist recorded the number of BMs detected per scan. One radiologist (V.N.) determined the scan on which BMs were most conspicuous by visual inspection. The 3 individual time-anonymized scans were loaded into the iPlan RT (Brainlab AG) server and had the window and level individually adjusted to permit clear distinction of the tumor margins from contiguous normal brain parenchyma. The perimeters of each lesion greater than $2 \mathrm{~mm}$ in diameter (the minimum size for accurate measurement) were contoured along the edges of visible enhancement for each slice on which the tumors were visualized for each contrast-enhanced sequence. Scan-specific individual tumor volumes were automatically calculated through interpolation by the iPlan software. There were no predefined "optimal" thresholding settings observed uniformly through the imaging and contouring process. When necessary to resolve fine detail, settings were ad- 
TABLE 1. Patient primary site of disease

\begin{tabular}{cc}
\hline Primary Site of Disease & No. of Patients \\
\hline Overall & 38 \\
\hline Breast & 6 \\
\hline Colon & 2 \\
\hline Lung & 23 \\
\hline Melanoma & 4 \\
\hline Ovarian & 1 \\
\hline Renal & 2 \\
\hline
\end{tabular}

justed. When later studies revealed lesions that had not been detected on earlier studies, the corresponding scans were rescrutinized for any evidence of overlooked lesions. Repetitive contouring was not performed to assess interand intraindividual variation in contouring.

\section{Statistical Analysis}

To analyze the differences in lesion count among the 3 imaging times, interclass correlations were established for the number of lesions counted by the radiologists to measure the interrater reliability, or agreement. The interclass correlations for the first scan (Scan 1), second scan (Scan 2), and third scan (Scan 3) were 0.7392, 0.7951, and 0.7290, respectively, demonstrating good interrater reliability. Given these findings, the number of lesions counted by the senior neuroradiologist was used for all subsequent analyses.

A Wilcoxon signed-rank test was used to determine whether the difference between the number of detected metastases on the first scan (immediately after contrast injection), as compared with scans at 10- and 15-minute delays, was significant. Accordingly, the proportion of subjects in whom at least 1 additional tumor was detected at each of the 3 successive testing stages was calculated, along with their corresponding exact $90 \%$ binomial CIs.

To analyze changes in lesion volume at each successive imaging time, a linear mixed model was used. Percent change in volume for each lesion was expressed as the log ratio of tumor volume at a later scan relative to the one before it. The significance level was Bonferroni-adjusted for 2 primary outcomes, which are percent change from 1) the immediate scan to the scan at a 10-minute delay, and 2) the scan at 10-minute delay to the scan at 15-minute delay. Results were considered significant at the level of $p$ $<0.025(0.05 \div 2=0.025)$.

Because some tumors were not visible on all 3 scans, calculating their volume change was a challenge. Clearly, if a lesion was not seen on an early scan but became visible at a later imaging time, it could not have had a volume of 0 during the earlier scan. However, because it was not actually seen, it was impossible to calculate its initial volume. Thus, for statistical purposes, we had to assign a superficial "lower measurable limit" to those tumors. Thus, "treatable" tumors were defined as tumors that were enhanced, visible, and measurable ( $>2 \mathrm{~mm}$ in diameter) in at least 1 of the 3 scans. A tumor that was seen in a later scan but was not enhanced, visible, or measurable in an earlier scan was given a volume of $0.001 \mathrm{~mm}^{3}$ in the earlier scan, which we defined for this study as a lower bound of "mea- surable" volume. One data outlier was removed because of this adjustment. Removal from the study of all tumors that were not visible on each of the 3 scans would skew the analysis, because many tumors that clearly appeared on later scans were not visible at earlier imaging times.

\section{Results \\ Lesion Number}

In $35.3 \%$ of all subjects (95\% CI $22.4 \%-49.9 \%)$, at least 1 new lesion was detected in the second scan as compared with the first scan. The range of increase in the number of metastases detected was $1-10$, with a mode of 1 . An additional $21.6 \%$ of subjects (95\% CI $11.3 \%-35.3 \%$ ) had at least 1 new lesion detected in the third scan as compared with the second scan. The range was $1-9$, with a mode of 1 metastasis (Table 2).

\section{Lesion Conspicuity}

In 34 of the 51 subjects (66.7\%), the radiologist blindly selected the third scan (acquired 15 minutes after injection) as the one providing the best lesion visibility and conspicuity. The second scan was selected as having the best conspicuity in $25.5 \%$ of subjects, and the first scan was selected in $7.8 \%$ of subjects.

Additionally, it was found that $21.6 \%$ (95\% CI $11.3 \%-$ $35.3 \%$ ) of patients had a decrease in the number of lesions detected between Scans 1 and 2, and 15.7\% (95\% CI $7.0 \%-28.6 \%$ ) had a decrease in the number of lesions detected between Scans 2 and 3. The range of decrease for both comparisons was from 1 to 3 , with a mode of 1 lesion. It is important to note that none of the lesions that disappeared were large enough to be measured accurately $(<2 \mathrm{~mm}$ in diameter). At this size, it is probable that the "lesions" were vascular enhancements rather than metastases, and thus would not have been treated. To ensure that there was an overall increase in the number of lesions seen at a time delay, the difference in tumor number between Scans 1 and 3 was also compared. There was at least 1 new lesion detected in the third scan as compared with the first scan in $43.1 \%$ (95\% CI 29.3\%-57.8\%) of subjects, indicating an overall increase in the number of metastases seen between the immediate and delayed scans. The increase in the number of metastases between Scans 1 and 2 and between Scans 1 and 3 was found to be statistically significant $(\mathrm{p}=0.0367$ and $\mathrm{p}=0.0264$, respectively).

\section{Lesion Volume}

There were a total of 234 treatable lesions among 49 subjects who underwent delayed-time MRI for the detec-

TABLE 2. Lesion number increase with delayed MRI

\begin{tabular}{lccc}
\hline \multicolumn{1}{c}{ Parameter } & Scan 1:2 & Scan 2:3 & Scan 1:3 \\
\hline $\begin{array}{c}\% \text { of studies w/ } \\
\geq 1 \text { new le- }\end{array}$ & $35.3(p=0.0367)$ & $21.6(p=0.273)$ & $43.1(p=0.0264)$ \\
sion & & & \\
\hline $95 \% \mathrm{Cl}$ & $22.4 \%-49.9 \%$ & $11.3 \%-35.3 \%$ & $29.3 \%-57.8 \%$ \\
\hline $\begin{array}{l}\text { Range of in- } \\
\text { crease }\end{array}$ & $1-10$ & $1-9$ & $1-14$ \\
\hline
\end{tabular}


tion of BMs. There was a statistically significant increase of $25.37 \%$ in the tumor volume detected from Scans 1 to $2(\mathrm{p}<0.0001)$. There was no significant increase in the tumor volume detected from Scans 2 to 3 ( $\mathrm{p}<0.4818$ ). However, 1 lesion was visualized on Scan 2 with a measurable volume of $0.045 \mathrm{~mm}^{3}$ that was not visualized on Scan 3, which resulted in an assigned lower measurement bound of $0.001 \mathrm{~mm}^{3}$. This was an uncommon occurrence, which resulted in that tumor being an outlier. When this 1 lesion was removed from the analysis, there was a significant increase in tumor volume detected from Scans 2 to 3 $(\mathrm{p}<0.0001)$ (Table 3).

\section{Time-Delayed Contrast-Enhanced MRI}

Detection of lesions after a time delay is illustrated in Figs. 1-3.

\section{Discussion}

Stereotactic radiosurgery of BMs is inherently an image-based technique. For this reason, imaging should accurately quantify the number of BMs, maximize the conspicuity of these lesions, and provide reliable lesion morphology to contour lesions to be targeted by imageguided treatment. In this study, we prospectively analyzed the effects of time delay on the detection of BMs. Improving metastasis detection prior to an initial treatment is necessary to avoid the selection of inappropriate interventions.

For example, if a patient has an ostensibly single, $2-\mathrm{cm}$ brain metastasis without significant mass effect or clinical symptoms, it is potentially treatable with either resection or SRS. If the patient actually has several additional small CNS metastases that are revealed by more sensitive MRI, surgery may no longer be a first choice for management. Moreover, if such a patient's management is SRS alone (to avoid neurocognitive sequelae) and only the largest, index metastasis is treated, then the omission of wholebrain irradiation or focal treatment with SRS of the smallest metastases would almost certainly soon be followed by the manifestation of additional metastatic foci on routine post-SRS surveillance imaging. The need in these cases for salvage therapy adds to the cost of patient management and is distressing to the patient and caregivers alike. Finally, timely identification of BMs is highly cost effective, helping to prevent unnecessary surgeries and aggressive radiation therapy for lesions that could have been treated earlier, at a lower cost and with a lower morbidity rate. ${ }^{16}$

To optimize lesion conspicuity, gadobenate dimeglumine was selected for this study because it has been shown to have a higher contrast-to-noise ratio compared with other gadolinium-based agents. ${ }^{24}$ Patients were examined using thin-section imaging (0.9-1.0-mm slice thickness, which increases the sensitivity of lesion detection ${ }^{6,17}$ ), and isotropic imaging data are needed for SRS treatment planning for reliable morphological contouring.

We found more lesions with a time delay after contrast injection. Of patients in our study, 35.3\% showed at least 1 new lesion when imaged 10 minutes after injection. In addition, there was a $25.4 \%$ mean increase in metastasis volume between immediate imaging and that done at a 10-minute delay. There was no significant increase in the
TABLE 3. Lesion volume changes with delayed MRI

\begin{tabular}{ccc}
\hline Scan & Median Percent Change & \multicolumn{1}{c}{ Q1, Q3* } \\
\hline Scan 2-Scan 1 & 25.37 & $10.98,50.34$ \\
\hline Scan 3-Scan 2 & 9.00 & $1.92,25.25$ \\
\hline * Q1 = 25th percentile or first quartile; $\mathbf{Q}$ = 75th percentile or third quartile.
\end{tabular}

number of metastases seen between the scans performed at 10- and 15-minute delays. However, there was a statistically significant $(9 \%)$ increase in volume between the scans at 10 and 15 minutes. The scan acquired 15 minutes after injection provided the best tumor conspicuity in $66.7 \%$ of those studied. Therefore, we conclude that peak enhancement was achieved during the window of 10-15 minutes after injection. This is effective for improved lesion enhancement and still provides enough flexibility for the clinical setting.

The clinical implications of the increase in metastasis volume over time may be significant. It is known that for $\mathrm{BMs}$, there is often an extension of microscopic disease beyond the radiographically detected tumor. ${ }^{3}$ A nonrandomized, single-institution retrospective study reported improved local control after SRS when a 1-mm margin was added to the contrast-enhanced tumor. ${ }^{19}$ The possibility exists that by using a delayed acquisition to include "all" of the metastases within the SRS prescription-isodose surface, local control with SRS alone may be maximized.

Delayed imaging with other gadolinium-based agents has previously shown improved lesion detection. Schörner et al. found that after an 8.5-minute delay, tumor-signal intensity increased. In addition, they noted that central tumor necrosis exhibited a delayed uptake of contrast. ${ }^{23}$ Yuh et al. found that detection of lesions smaller than $10 \mathrm{~mm}$ improved after both a 10- and 20-minute delay. ${ }^{35}$ However, the increased relaxivity of gadobenate dimeglumine allows it to be used with shorter delay times, making it more efficient in a busy clinical imaging setting.

Successful imaging relies on ascertaining how closely enhancement on MR images correlates to the tumor's actual physiology. The leptomeningeal enhancement caused by BMs on MR images is due to a disruption of the bloodbrain barrier, because metastases have an abnormally high capillary permeability compared with healthy tissue. Gadobenate dimeglumine interacts with serum albumin. This increases $\mathrm{R} 1$ relaxivity, which results in brighter, more avid contrast enhancement. A delay in imaging may be effective in increasing contrast intensity because it allows more time for aberrant and leaky neovasculature within the metastasis to be perfused with the contrast. ${ }^{15}$ The small vascular surface of tiny metastatic foci may not permit very much contrast to be extravasated with firstpass kinetics. Additional diffusion of contrast from the neovasculature almost certainly occurs over time as contrast recirculates through the cerebral vasculature.

One possible limitation to our study is that glucocorticoid analogs, such as dexamethasone, were administered to our patients for the treatment of increased intracranial pressure due to tumor mass effect. It is known that glucocorticoid analogs cause significantly decreased T1 signal uptake, decreasing enhancement. ${ }^{33}$ In our study, all but 3 patients were treated with either intravenous or oral dexa- 

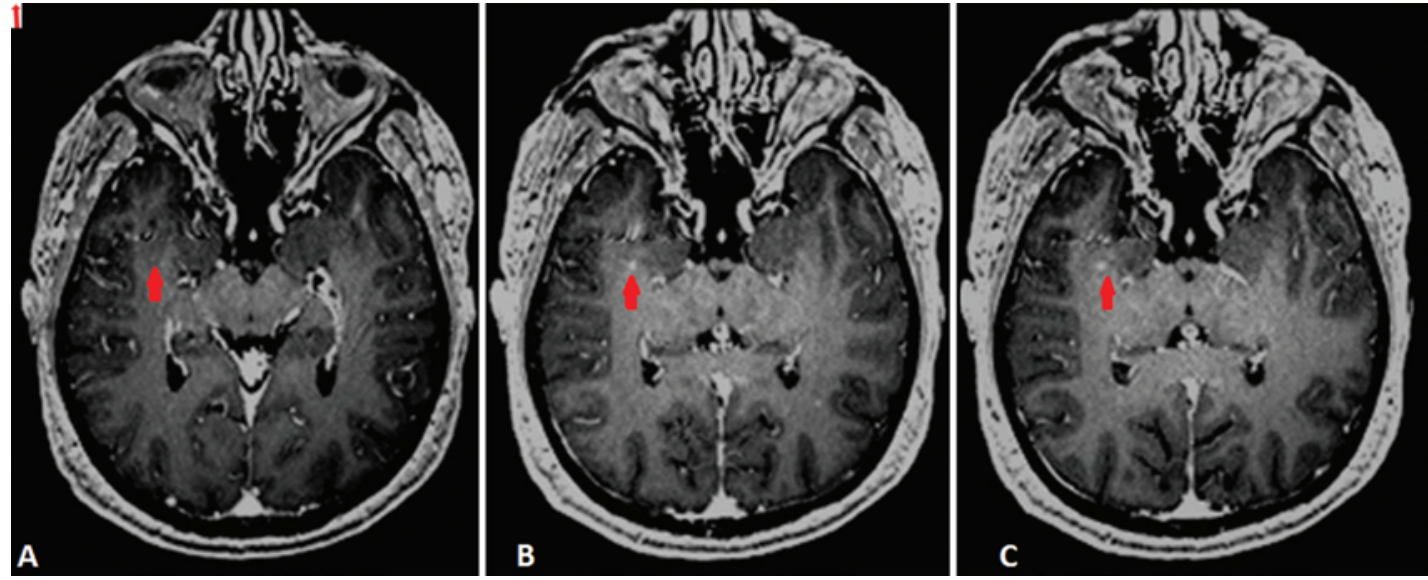

FIG. 1. In a study performed immediately after contrast injection (A), there is no lesion visible in the right parahippocampal region. However, a treatable tumor becomes visible at 10-minute (B) and 15-minute (C) delays. Red arrows denote where a lesion is not visualized $(\mathbf{A})$ and where it becomes manifest ( $B$ and $\mathbf{C})$. Figure is available in color online only.

methasone at the time of imaging. Because dexamethasone causes blood vessels to be less permeable to contrast, our results may have shown fewer lesions, with less contrast perfusion, than were actually present. Although the results of our study were probably not significantly skewed, the administration of dexamethasone prior to imaging may be an issue that is common to many patients who are being imaged to plan for SRS.

An additional limitation to the study was difficulty in delineating between true tumor enhancement and the leakage of contrast into edematous areas surrounding the lesions. There is currently no correction formula or imaging technique that clearly distinguishes this "pseudoenhancement" from contrast-enhanced lesions. The application of dynamic contrast-enhanced MRI to evaluate leakage of contrast in surrounding tissues was not feasible in this study. Without a biopsy of the tumor margins, there is no way to definitively determine whether the enhanced tissue is truly malignant. Thus, it is possible that some of the tumor growth we saw may have actually been contrast leakage. Clinically, it is better to irradiate areas of edema surrounding BMs than to undertreat the tumors themselves and risk a local recurrence. However, in the future, it would be valuable to establish a correction formula for BM volume, taking edema into account. High-dose gadolinium has been documented to be another method of improving the detection of small BMs.$^{35}$ However, using a time delay may be superior to higher contrast dose for several reasons. First, the benefits for tumor detection have been inconclusive thus far., ${ }^{7,27}$ Furthermore, using more contrast may increase the risk of nephrogenic systemic fibrosis for some patients, and using more contrast increases costs. A direct comparison of multiple-dose contrast and delayed imaging would be helpful to determine if one method is superior for metastasis detection.

\section{Conclusions}

The importance of timely detection of BMs has been demonstrated for cost effectiveness, treatment success, and, most importantly, patient comfort. Our study showed that lesion number, volume, and conspicuity increase with a time delay following the administration of gadobenate dimeglumine on both 1.5-T and 3-T magnets. We recommend that postcontrast MR images be acquired between 10 and 15 minutes after injection for peak tumor defini-
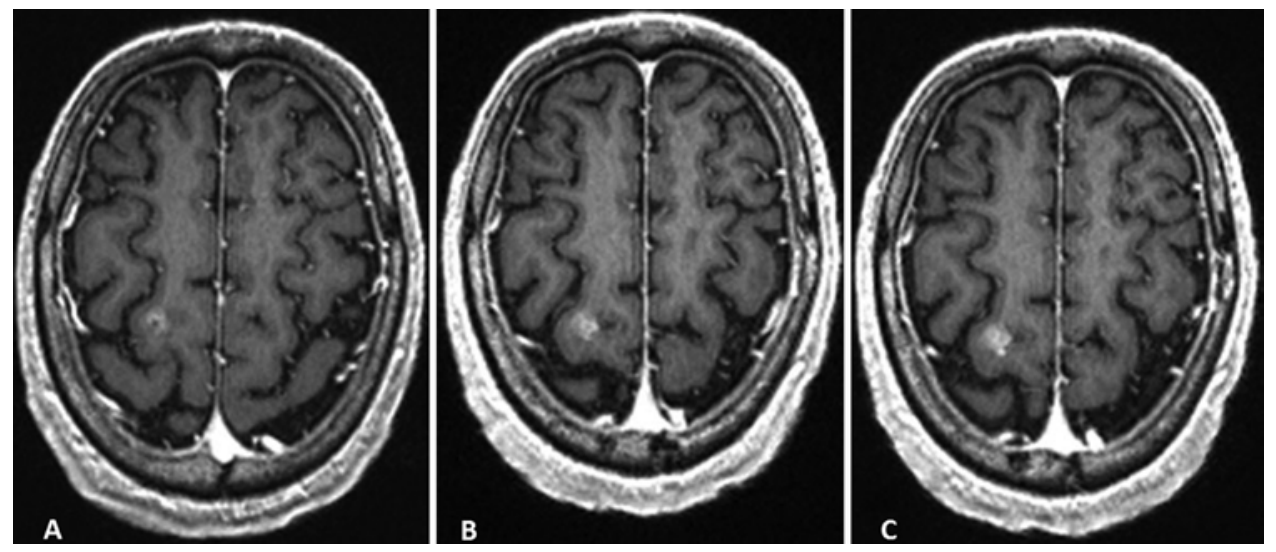

FIG. 2. In a study performed immediately after contrast injection (A), there is some enhancement. At a delay (B and C), there is more avid enhancement, more confluent central enhancement, and the lesion margins are clearer. 

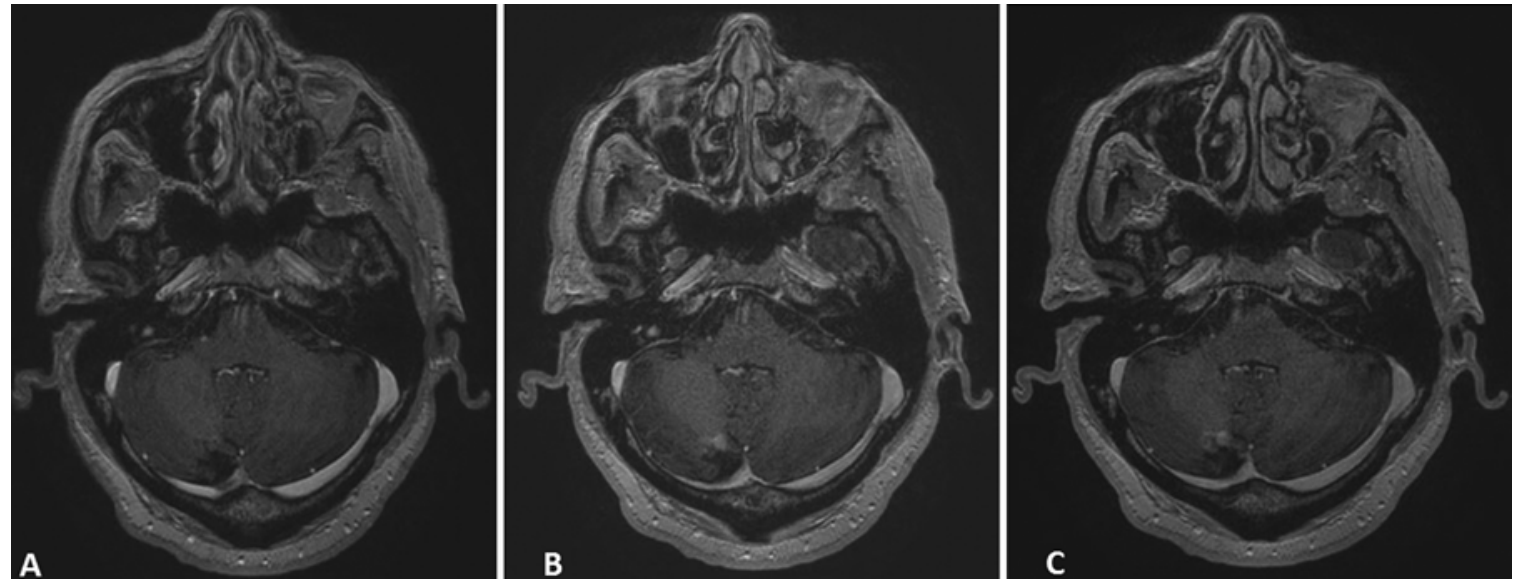

FIG. 3. Increased apparent tumor volume after delayed imaging allows for improved treatment planning. In this resection cavity, imaging immediately after contrast injection showed no enhancement (A). At 10-minute (B) and 15-minute (C) delays, there is additional enhancement visible, possibly suggesting an unresected tumor.

tion. Volumetric, delayed gadolinium-enhanced imaging has become our institutional standard for BMs, regardless of whether SRS is planned.

\section{Acknowledgments}

We thank Elaine Montchal for patient recruitment and excellent patient care, as well as Jonathan Pun for assistance with the preparation of images for our manuscript.

\section{References}

1. Alexander E III, Moriarty TM, Davis RB, Wen PY, Fine HA, Black PM, et al: Stereotactic radiosurgery for the definitive, noninvasive treatment of brain metastases. J Natl Cancer Inst 87:34-40, 1995

2. Aoyama H, Shirato H, Tago M, Nakagawa K, Toyoda T, Hatano K, et al: Stereotactic radiosurgery plus whole-brain radiation therapy vs stereotactic radiosurgery alone for treatment of brain metastases: a randomized controlled trial. JAMA 295:2483-2491, 2006

3. Baumert BG, Rutten I, Dehing-Oberije C, Twijnstra A, Dirx MJ, Debougnoux-Huppertz RM, et al: A pathology-based substrate for target definition in radiosurgery of brain metastases. Int J Radiat Oncol Biol Phys 66:187-194, 2006

4. Chang EL, Wefel JS, Hess KR, Allen PK, Lang FF, Kornguth DG, et al: Neurocognition in patients with brain metastases treated with radiosurgery or radiosurgery plus whole-brain irradiation: a randomised controlled trial. Lancet Oncol 10:1037-1044, 2009

5. Do L, Pezner R, Radany E, Liu A, Staud C, Badie B: Resection followed by stereotactic radiosurgery to resection cavity for intracranial metastases. Int J Radiat Oncol Biol Phys 73:486-491, 2009

6. Donahue BR, Goldberg JD, Golfinos JG, Knopp EA, Comiskey J, Rush SC, et al: Importance of MR technique for stereotactic radiosurgery. Neuro Oncol 5:268-274, 2003

7. Filippi M, Yousry T, Rocca MA, Fesl G, Voltz R, Comi G: Sensitivity of delayed gadolinium-enhanced MRI in multiple sclerosis. Acta Neurol Scand 95:331-334, 1997

8. Gaspar LE, Gutin PH, Rogers L, Schneider JF, Larson D, Bloomer WD, et al: Pre-irradiation evaluation and management of brain metastases. American College of Radiology. ACR Appropriateness Criteria. Radiology 215 Suppl:1105-1110, 2000

9. Jawahar A, Jawahar LL, Nanda A, Sharp CD, Warren A,
Elrod JW, et al: Stereotactic radiosurgery using the Leksell Gamma Knife: current trends and future directives. Front Biosci 9:932-938, 2004

10. Jenkinson MD, Haylock B, Shenoy A, Husband D, Javadpour M: Management of cerebral metastasis: evidence-based approach for surgery, stereotactic radiosurgery and radiotherapy. Eur J Cancer 47:649-655, 2011

11. Karnofsky D, Burchenal JH: The clinical evaluation of chemotherapeutic agents in cancer, in MacLeod CM (ed): Evaluation of Chemotherapeutic Agents. New York: Columbia University Press, 1949, pp 191-205

12. Kocher M, Soffietti R, Abacioglu U, Villà S, Fauchon F, Baumert BG, et al: Adjuvant whole-brain radiotherapy versus observation after radiosurgery or surgical resection of one to three cerebral metastases: results of the EORTC 2295226001 study. J Clin Oncol 29:134-141, 2011

13. Kuo PH, Kanal E, Abu-Alfa AK, Cowper SE: Gadoliniumbased MR contrast agents and nephrogenic systemic fibrosis. Radiology 242:647-649, 2007

14. Lu JJ, Brady LW: Decision Making in Radiation Oncology. Berlin: Springer, 2011

15. Lüdemann L, Grieger W, Wurm R, Wust P, Zimmer C: Quantitative measurement of leakage volume and permeability in gliomas, meningiomas and brain metastases with dynamic contrast-enhanced MRI. Magn Reson Imaging 23:833-841, 2005

16. Mayr NA, Yuh WT, Muhonen MG, Fisher DJ, Nguyen HD, Ehrhardt JC, et al: Cost-effectiveness of high-dose MR contrast studies in the evaluation of brain metastases. AJNR Am J Neuroradiol 15:1053-1061, 1994

17. Nagai A, Shibamoto Y, Mori Y, Hashizume C, Hagiwara M, Kobayashi $\mathrm{T}$ : Increases in the number of brain metastases detected at frame-fixed, thin-slice MRI for gamma knife surgery planning. Neuro Oncol 12:1187-1192, 2010

18. National Comprehensive Cancer Network: NCCN Clinical Practice Guidelines in Oncology: Central Nervous System Cancers (Version 1.2015). Fort Washington, PA: National Comprehensive Cancer Network, 2015

19. Noël G, Simon JM, Valery CA, Cornu P, Boisserie G, Hasboun D, et al: Radiosurgery for brain metastasis: impact of CTV on local control. Radiother Oncol 68:15-21, 2003

20. Picozzi P, Kirchin MA: Improving lesion detection and visualization: implications for neurosurgical planning and followup. Neuroradiology 49 (Suppl 1):S27-S34, 2007

21. Rowley HA, Scialfa G, Gao PY, Maldjian JA, Hassell D, Kuhn MJ, et al: Contrast-enhanced MR imaging of brain lesions: a large-scale intraindividual crossover comparison of 
gadobenate dimeglumine versus gadodiamide. AJNR Am J Neuroradiol 29:1684-1691, 2008

22. Schellinger PD, Meinck HM, Thron A: Diagnostic accuracy of MRI compared to CCT in patients with brain metastases. J Neurooncol 44:275-281, 1999

23. Schörner W, Laniado M, Niendorf HP, Schubert C, Felix R: Time-dependent changes in image contrast in brain tumors after gadolinium-DTPA. AJNR Am J Neuroradiol 7:10131020,1986

24. Silver NC, Good CD, Barker GJ, MacManus DG, Thompson AJ, Moseley IF, et al: Sensitivity of contrast enhanced MRI in multiple sclerosis. Effects of gadolinium dose, magnetization transfer contrast and delayed imaging. Brain 120:11491161, 1997

25. Soffietti R, Cornu P, Delattre JY, Grant R, Graus F, Grisold $\mathrm{W}$, et al: EFNS Guidelines on diagnosis and treatment of brain metastases: report of an EFNS Task Force. Eur J Neurol 13:674-681, 2006

26. Soffietti R, Rudā R, Mutani R: Management of brain metastases. J Neurol 249:1357-1369, 2002

27. Sze G, Johnson C, Kawamura Y, Goldberg SN, Lange R, Friedland RJ, et al: Comparison of single- and triple-dose contrast material in the MR screening of brain metastases. AJNR Am J Neuroradiol 19:821-828, 1998

28. Tsao MN, Lloyd NS, Wong RK: Clinical practice guideline on the optimal radiotherapeutic management of brain metastases. BMC Cancer 5:34, 2005

29. Tsao MN, Rades D, Wirth A, Lo SS, Danielson BL, Gaspar LE, et al: Radiotherapeutic and surgical management for newly diagnosed brain metastasis(es): An American Society for Radiation Oncology evidence-based guideline. Pract Radiat Oncol 2:210-225, 2012

30. Van Dijk P, Sijens PE, Schmitz PIM, Oudkerk M: Gdenhanced MR imaging of brain metastases: contrast as a function of dose and lesion size. Magn Reson Imaging 15:535-541, 1997

31. Wen PY, Loeffler JS: Brain metastases. Curr Treat Options Oncol 1:447-458, 2000

32. Wen PY, Loeffler JS: Management of brain metastases. Oncology (Williston Park) 13:941-954, 957-962, 969, 1999

33. Wilkinson ID, Jellineck DA, Levy D, Giesel FL, Romanowski CA, Miller BA, et al: Dexamethasone and enhancing solitary cerebral mass lesions: alterations in perfusion and blood-tumor barrier kinetics shown by magnetic resonance imaging. Neurosurgery 58:640-646, 2006
34. Yamamoto M, Serizawa T, Shuto T, Akabane A, Higuchi Y, Kawagishi J, et al: Stereotactic radiosurgery for patients with multiple brain metastases (JLGK0901): a multi-institutional prospective observational study. Lancet Oncol 15:387-395, 2014

35. Yuh WT, Tali ET, Nguyen HD, Simonson TM, Mayr NA, Fisher DJ: The effect of contrast dose, imaging time, and lesion size in the MR detection of intracerebral metastasis. AJNR Am J Neuroradiol 16:373-380, 1995

\section{Disclosure}

The authors report no conflict of interest concerning the materials or methods used in this study or the findings specified in this paper.

\section{Supplemental Information}

Previous Presentation

Portions of this work's abstract were presented orally at the International Stereotactic Radiosurgery Society Congress, June 16, 2013; American Society of Neuroradiology Annual Meeting, May 21, 2013; Quadrennial Meeting of the World Society of Stereotactic and Functional Neurosurgery, May 28, 2013; and American Society of Radiation Oncology Annual Meeting, September 24, 2013

\section{Author Contributions}

Conception and design: Knisely, Nguyen, Schulder. Acquisition of data: Knisely, Kushnirsky, Nguyen, Katz, Warshall. Analysis and interpretation of data: Nguyen, Katz, Steinklein, Rosen, Warshall. Drafting the article: Knisely, Kushnirsky. Critically revising the article: Knisely, Kushnirsky, Nguyen. Approved the final version of the manuscript on behalf of all authors: Knisely. Statistical analysis: Rosen. Administrative/technical/material support: Nguyen, Katz. Study supervision: Knisely, Nguyen, Schulder.

\section{Correspondence}

Jonathan Knisely, Department of Radiation Medicine, North Shore-LIJ Health System, Hofstra North Shore-LIJ School of Medicine at Hofstra University, 450 Lakeville Rd., Lake Success, NY 11042. email: jknisely@nshs.edu. 